\title{
Argatroban for therapeutic anticoagulation for heparin resistance associated with Covid-19 infection
}

\author{
Fergal McGlynn ${ }^{1}$ (D) Jennifer McGrath ${ }^{2} \cdot$ Chithra Varghese $^{3} \cdot$ Barbara Ryan $^{4} \cdot$ Johnny McHugh $^{1} \cdot$ Arabella Fahy $^{2}$. \\ Helen Enright ${ }^{1}$
}

Accepted: 10 August 2020 / Published online: 24 August 2020

○) Springer Science+Business Media, LLC, part of Springer Nature 2020

\section{Dear Editor,}

We read with interest the report of White et al. in Journal of Thrombosis and Thrombolysis [1]. They describe their experience with Covid-19 patients in the intensive care unit anti-coagulated with low molecular weight heparin (LMWH) and unfractionated heparin (UFH), presenting evidence of apparent heparin resistance (HR) resulting from increased levels of factor VIII and fibrinogen with decreased antithrombin levels. Recently Connors and Levy also discuss Covid-19 and its associated coagulopathy [2]. They recommend LMWH or UFH for therapeutic anticoagulation, for any indication, over direct oral anticoagulation (DOAC), and recommend considering measuring anti-Xa levels for anticoagulant monitoring.

Buen et al. reported 4 patients with Covid-19 infection with apparent HR (defined as the need for doses of UFH of $>35,000 \mathrm{IU} /$ day to achieve anticoagulation) [3]. These patients had raised factor VIII, fibrinogen and d-dimer levels, as with many Covid-19 patients. They point out the in vitro effect of increased factor VIII levels on heparin resulting in lower activated partial thromboplastin time (aPTT), i.e. apparent HR. This does not occur in vivo, leading to the risk of bleeding complications as heparin doses are increased. They also recommend measuring anti-Xa levels in patients with Covid-19 who are anticoagulated with heparin.

Fergal McGlynn

fergal.mcglynn@gmail.com

1 Department of Haematology, Tallaght University Hospital, Tallaght D24 NR0A, Ireland

2 Department of Intensive Care Medicine, Tallaght University Hospital, Tallaght D24 NROA, Ireland

3 Department of Respiratory Medicine, Tallaght University Hospital, Tallaght D24 NROA, Ireland

4 Department of Gastroenterology, Tallaght University Hospital, Tallaght D24 NROA, Ireland
Bachler et al. [4] recently reported outcomes for 42 patients in (non-Covid-19) critical care settings with HR who were anti-coagulated with argatroban compared to those treated with increased doses of UFH. They reported greater efficacy in achieving adequate anti-coagulation (as measured by the aPTT) at $24 \mathrm{~h}$ with argatroban than with increased doses of heparin. There was no significant difference in bleeding or thromboembolic events between the treatment arms.

We report 2 cases of apparent HR in critically ill Covid19 patients with thrombotic complications who were successfully and safely treated with argatroban.

\section{Case 1}

A 33 year old male with no significant medical history presented with a five day history of fever and worsening shortness of breath. Oxygen saturations were $98 \%$ on $10 \mathrm{~L}$ of oxygen via face mask. Full blood count showed lymphopenia $0.7 \times 10^{9} / \mathrm{L}$ (normal $1.50-4.99$ ); the platelet count was normal. C-reactive protein (CRP) was $72 \mathrm{mg} / \mathrm{L}$ (normal 0-5), serum ferritin was $658 \mu \mathrm{g} / \mathrm{L}$ (normal 14-200) and d-dimer $0.83 \mu \mathrm{g} / \mathrm{l}$ (normal 0-0.42). Prothrombin time (PT) was $10.7 \mathrm{~s}$ and aPTT was $23 \mathrm{~s}$, both normal. IL-6 $59 \mathrm{pg} / \mathrm{ml}$ (normal $0-7)$ and procalcitonin $0.208 \mathrm{ng} / \mathrm{ml}$ (normal $<0.1$ ) levels were subsequently found to be elevated. Chest X-ray showed extensive bilateral infiltrates. Covid-19 was confirmed by positive reverse transcriptase polymerase chain reaction (RT-PCR) assay for severe acute respiratory syndrome coronavirus 2 (SARS-CoV-2).

The patient was initially treated with ceftriaxone and hydroxychloroquine. Prophylactic enoxaparin was given (40 mg once daily). He required admission to the Intensive Treatment Unit (ITU) and intubation within $24 \mathrm{~h}$ due to progressive hypoxia. D-Dimer levels rose to $7.13 \mu \mathrm{g} / \mathrm{L}$. 
He developed recurrent arterial line clotting problems, requiring replacement of the line four times within $18 \mathrm{~h}$, and a catheter associated venous thrombosis of the internal jugular vein. Computed Tomography Pulmonary Angiogram (CTPA) subsequently demonstrated a segmental acute pulmonary embolism (PE) with pulmonary infarction, against a background of changes typical of SARS-CoV-2 infection.

Unfractionated heparin infusion was commenced. However, the patient's aPTT did not become prolonged despite dose titrations up to 2755 units per hour and remained within the normal range over $72 \mathrm{~h}$. Anti-thrombin level was $0.68 \mathrm{IU} /$ $\mathrm{mL}$ (normal $0.87-1.19$ ), factor VIII activity was markedly elevated at $4.77 \mathrm{IU} / \mathrm{mL}$ (normal 0.55-1.40) and fibrinogen was $6.76 \mathrm{~g} / \mathrm{L}$ (normal 1.50-4.00). Anti-Xa levels were not obtained. Anti-coagulation was switched to argatroban at a rate of $2 \mu \mathrm{g} / \mathrm{kg} / \mathrm{min}$ and therapeutic anti-coagulation, as indicated by the aPTT ratio greater than 1.5 , was achieved within five hours. Therapeutic anti-coagulation was maintained using argatroban for nine days without bleeding complications or further thrombotic events. Subsequently his condition slowly improved and he was eventually discharged.

\section{Case 2}

A 47 year old male presented to the Emergency Department with a 2 week history of dyspnea, dry cough, fever, myalgia, chills and anorexia. On arrival, his oxygen saturations were $89 \%$ on room air with associated tachypnoea. Full blood count showed lymphopenia $0.5 \times 10^{9} / \mathrm{L}$; the platelet count was normal. CRP was $212 \mathrm{mg} / \mathrm{L}$, serum ferritin $1887 \mu \mathrm{g} / \mathrm{L}$, d-dimer $1.25 \mu \mathrm{g} / \mathrm{L}$. IL-6 was $95.2 \mathrm{pg} / \mathrm{mL}$ and procalcitonin $0.604 \mathrm{ng} / \mathrm{mL}$. PT was $10.9 \mathrm{~s}$, aPTT $26.5 \mathrm{~s}$, both normal. Chest X-ray showed bilateral infiltrates. Covid-19 infection was confirmed by RT-PCR.

Over the course of a few hours he became increasingly hypoxic and required intubation and subsequently dialysis. By day 10 of his ITU admission his oxygen requirements remained high with a d-dimer level of $5.25 \mu \mathrm{g} / \mathrm{L}$. He was therefore empirically commenced on an intravenous heparin infusion, initially with an infusion dose of 9 units $/ \mathrm{kg} / \mathrm{h}$. Dose adjustments continued up to $16 \mathrm{units} / \mathrm{kg} / \mathrm{h}$ over $22 \mathrm{~h}$ but the aPTT failed to respond.

Persistent problems arose with clotting of his dialysis catheter. His anticoagulation was therefore changed to argatroban at a rate of $0.5 \mu \mathrm{g} / \mathrm{kg} / \mathrm{min}$ due to multi-organ dysfunction. Therapeutic anticoagulation, as indicated by aPTT ratio greater than 1.5 , was achieved within four hours of its commencement. There were no further issues arising from the dialysis filter. Due to the clinical suspicion of a pulmonary embolus, he was continued on systemic anticoagulation for a total of 14 days. As renal and liver function improved over time the argatroban infusion rate was titrated upwards to the maximum dose of $2 \mu \mathrm{g} /$ $\mathrm{kg} / \mathrm{min}$. There were no bleeding complications or further thrombotic episodes whilst on argatroban. The patient's clinical condition steadily improved and he was extubated following 25 days of mechanical ventilation.

\section{Discussion}

Argatroban has been shown to be an effective alternative treatment for anti-coagulation of patients in a critical care setting [4]. SARS-CoV-2 is a novel coronavirus leading to Covid-19 disease which necessitates critical care in $5 \%$ of cases [5]. Coagulopathy is a common feature of the disease. Some centres have noted HR in Covid-19 patients in ITU with coagulopathy, attributed to increased factor VIII levels, heparin-binding pro-inflammatory proteins, acute phase reactants such as pro-calcitonin and IL-6, and low ATIII levels [1, 3].

We present 2 cases of apparent heparin resistance in critically ill Covid-19 patients. Apparent heparin resistance is understood to be caused by the effect of increased factor VIII levels on heparin in vitro, lowering anticoagulant activity and hence interfering with aPTT measurement. This likely does not occur in vivo, leading to the risk of bleeding complications due to over-anticoagulation [2]. Both our patients had significant coagulopathy with thrombotic complications, and were apparently resistant to UFH, although anti-Xa levels were not obtained. Given the novel nature of Covid-19 disease, combined with the paucity of data available, but a strong impression of a prothrombotic inflammatory state, the possibility of genuine heparin resistance as a feature of this disease is raised.

Measuring anti-Xa levels in patients with Covid-19 who are anticoagulated with heparin may provide a solution and help determine whether a patient with apparent HR is, in fact, adequately anticoagulated. However, anti-Xa measurement is costly and may not be universally available out of hours. Furthermore, timing of blood sampling is critical for anti-Xa measurement for patients on LMWH, which can be challenging given the extra burden involved in caring for patients with Covid-19.

The use of an alternative anticoagulant should also be considered [4, 6]. We have observed two cases of HR in our ITU, both treated successfully and safely with argatroban for thrombotic complications. Argatroban may be considered as an alternative anti-coagulant, easily monitored using the aPTT in critically ill patients with Covid19-associated thrombosis and HR. 
Authors' contributions F.M. and J.M. wrote the document. C.V., B.R. and A.F. were the treating consultants and reviewed the document. J.M. and H.E. formulated the idea and reviewed the document.

Funding None received.

\section{Compliance with ethical standards}

Informed consent Informed consent was obtained from both patients.

\section{References}

1. White D, MacDonald S, Bull T et al (2020) Heparin resistance in COVID-19 patients in the intensive care unit. J Thromb Thrombol. https://doi.org/10.1007/s11239-020-02145-0

2. Connors JM, Levy JH (2020) COIVD-19 and its implications for thrombosis and anticoagulation. Blood. https://doi.org/10.1182/ blood.2020006000
3. Beun R et al (2020) Thromboembolic events and apparent heparin resistance in patients infected with SARS-CoV-2. Int J Lab Hematol. https://doi.org/10.1111/ijlh.13230

4. Bachler $\mathrm{M}$ et al (2020) A prospective pilot trial to assess the efficacy of argatroban (Argatra $\left.{ }^{\circledR}\right)$ in critically Ill patients with heparin resistance. J Clin Med 9(4):E963. https://doi.org/10.3390/ jcm9040963

5. Guan WJ, Ni ZY, Hu Y et al (2020) Clinical characteristics of coronavirus disease 2019 in China. N Engl J Med 382(18):17081720. https://doi.org/10.1056/NEJMoa2002032

6. Treichl B et al (2015) Efficacy of argatroban in critically ill patients with heparin resistance: a retrospective analysis. Semin Thromb Hemost 41(1):61-67. https://doi.org/10.1055/s-0034-1398382

Publisher's Note Springer Nature remains neutral with regard to jurisdictional claims in published maps and institutional affiliations. 